\title{
Multiple CO lines in SMM J16359+6612 - further evidence for a merger
}

\author{
A. Weiß ${ }^{1,4}$, D. Downes ${ }^{2}$, F. Walter ${ }^{3}$, and C. Henkel ${ }^{4}$ \\ 1 IRAM, Avenida Divina Pastora 7, 18012 Granada, Spain \\ e-mail: aweiss@iram.es \\ 2 IRAM, 300 rue de la Piscine, 38406 St.-Martin-d'Hères, France \\ 3 MPIA, Königstuhl 17, 69117 Heidelberg, Germany \\ 4 MPIfR, Auf dem Hügel 69, 53121 Bonn, Germany
}

Received 8 June 2005 / Accepted 29 July 2005

\begin{abstract}
Using the IRAM $30 \mathrm{~m}$ telescope, we report the detection of the $\mathrm{CO}(3-2), \mathrm{CO}(4-3), \mathrm{CO}(5-4)$ and $\mathrm{CO}(6-5)$ lines in the gravitational lensed submm galaxy SMM J16359+6612 at $z=2.5$. The CO lines have a double peak profile in all transitions. From a Gaussian decomposition of the spectra we show that the $\mathrm{CO}$ line ratios, and therefore the underlying physical conditions of the gas, are similar for the blue and the redshifted component. The CO line Spectral Energy Distribution (SED; i.e. flux density vs. rotational quantum number) turns over already at the CO 5-4 transition which shows that the molecular gas is less excited than in nearby starburst galaxies and high- $z$ QSOs. This difference mainly arises from a lower average $\mathrm{H}_{2}$ density, which indicates that the gas is less centrally concentrated than in nuclear starburst regions in local galaxies. We suggest that the bulk of the molecular gas in SMM J16359+6612 may arise from an overlap region of two merging galaxies. The low gas density and clear velocity separation may reflect an evolutionary stage of the merger event that is in between those seen in the Antennae and in the more evolved ultraluminous infrared galaxies (ULIRGs) like e.g. Mrk 231.
\end{abstract}

Key words. galaxies: formation - galaxies: high-redshift - galaxies: ISM - galaxies: individual (SMM J16359+6612) cosmology: observations

\section{Introduction}

The intensity of the far-infrared (FIR) background indicates that emission from warm dust contributes significantly to galaxies' overall energy output over the Hubble time (Puget et al. 1996; Fixsen et al. 1998). Recent surveys at submm and $\mathrm{mm}$ wavelengths with SCUBA at the JCMT and MAMBO at the IRAM $30 \mathrm{~m}$ telescope have identified part of the underlying galaxy population responsible for the strong FIR emission. Up to now several hundred submm/mm galaxies (SMGs) have been identified (e.g. Smail et al. 1997; Bertoldi et al. 2000; Ivison et al. 2002; Webb et al. 2003; Greve et al. 2004). These surveys, however, are limited by confusion and therefore only trace the bright part of the SMG luminosity function (Blain et al. 2002). Observations of $\mathrm{CO}$, providing valuable information on the dynamics, size and mass of the molecular reservoirs in these objects, have only been reported for 12 SMGs so far (e.g. Frayer et al. 1998; Ivison et al. 2001; Downes \& Solomon 2003; Genzel et al. 2003; Neri et al. 2003; Greve et al. 2005). Due to their selection based on the $\mathrm{mm} / \mathrm{submm}$ continuum, all these sources, however, are intrinsically luminous, and not representative of the faint end of the SMG luminosity function that dominates the FIR background.
Recently Kneib et al. (2004) discovered the strongly lensed submillimeter galaxy SMM J16359+6612 towards the galaxy cluster A 2218. This source is lensed by the cluster into 3 discrete images and the large magnification $(14,22$ and 9 for images $\mathrm{A}, \mathrm{B}$ and $\mathrm{C}$ respectively) implies that its intrinsic submm flux density $\left(S_{850 \mu \mathrm{m}}=0.8 \mathrm{mJy}\right.$, corresponding to $L_{\mathrm{FIR}} \approx$ $10^{12} L_{\odot}$, Kneib et al. 2004) is below the confusion limit of existing $850 \mu \mathrm{m}$ surveys ( $\approx 2 \mathrm{mJy}$, Blain et al. 2002). It therefore provides a unique opportunity to investigate a source which is presumably more representative for the submm population.

Detections of CO towards SMM J16359+6612 have been reported by Sheth et al. (2004, CO 3-2) and Kneib et al. (2005, CO 3-2 and 7-6). In this letter we report on the detection of the CO 3-2, 4-3, 5-4 and 6-5 lines towards the strongest lensed component $\mathrm{B}$. For the derived quantities in this paper, we use a $\Lambda$ cosmology with $H_{0}=71 \mathrm{~km} \mathrm{~s}^{-1} \mathrm{Mpc}^{-1}, \Omega_{\Lambda}=0.73$ and $\Omega_{\mathrm{m}}=0.27$ (Spergel et al. 2003).

\section{Observations}

Observations towards SMM J16359+6612 B were made with the IRAM $30 \mathrm{~m}$ telescope during nine runs between Jan. and March 2005 in mostly excellent weather conditions. We used the $\mathrm{AB}$ and $\mathrm{CD}$ receiver configuration with the $\mathrm{A} / \mathrm{B}$ receivers 

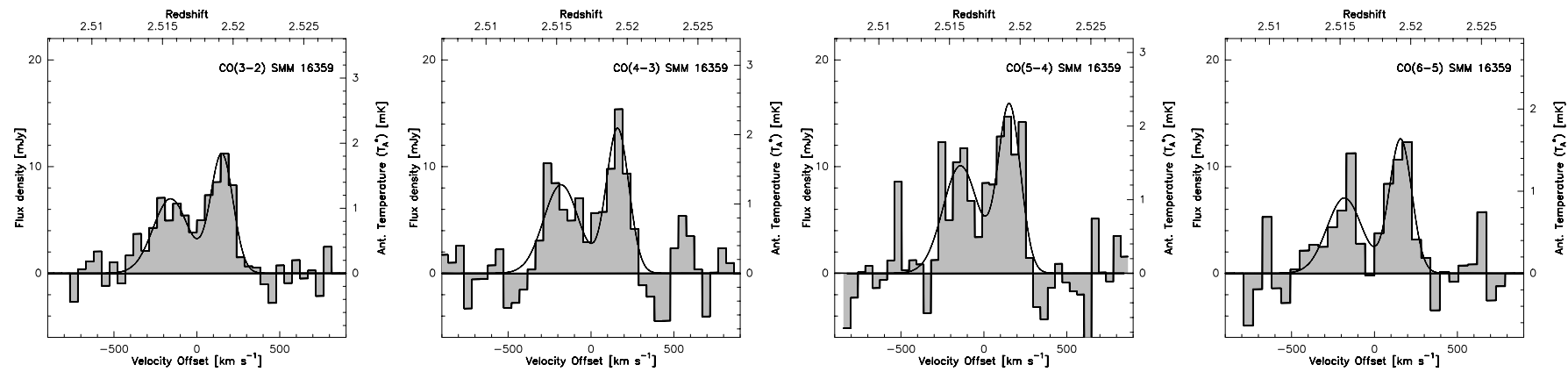

Fig. 1. Spectra of the $\mathrm{CO}(3-2), \mathrm{CO}(4-3), \mathrm{CO}(5-4)$ and $\mathrm{CO}(6-5)$ lines towards SMM J16359+6612 B, with Gaussian fit profiles superposed. The velocity scale is relative to a CO redshift of $z=2.5174$. The velocity resolution is $50 \mathrm{~km} \mathrm{~s}^{-1}(3-2,4-3,5-4)$ and $55 \mathrm{~km} \mathrm{~s}(6-5)$. All spectra are shown with the same flux scale.

tuned to the $\mathrm{CO}(3-2)(98.310 \mathrm{GHz}, 3 \mathrm{~mm}$ band) and $\mathrm{CO}(6-5)$ (196.586 GHz $1 \mathrm{~mm}$ band) and $\mathrm{C} / \mathrm{D}$ to $\mathrm{CO}(4-3)$ or $\mathrm{CO}(5-4)$ (131.074, 161.637GHz, $2 \mathrm{~mm}$ band). The beam sizes/antenna gains for increasing observing frequencies are $25^{\prime \prime} / 6.1 \mathrm{Jy} \mathrm{K}^{-1}$, $19^{\prime \prime} / 6.5 \mathrm{Jy} \mathrm{K}^{-1}, 15^{\prime \prime} / 6.9 \mathrm{Jy} \mathrm{K}^{-1}$ and $12.5^{\prime \prime} / 7.7 \mathrm{Jy} \mathrm{K}^{-1}$. Typical system temperatures were $\approx 120 \mathrm{~K}, 220 \mathrm{~K}$, and $270 \mathrm{~K}\left(T_{\mathrm{A}}^{*}\right)$ for the 3,2 , and $1 \mathrm{~mm}$ observations. The observations were done in wobbler switching mode, with switching frequencies of $0.5 \mathrm{~Hz}$ and a wobbler throw of $50^{\prime \prime}$ in azimuth. Pointing was checked frequently and was found to be stable to within $3^{\prime \prime}$. Calibration was done every $12 \mathrm{~min}$ using the standard hot/cold-load absorber measurements. The planets Uranus and Neptune were used for absolute flux calibration. We estimate the flux density scale to be accurate to about $\pm 10-15 \%$.

Data were taken with the $1 \mathrm{MHz}$ filter banks on the A/B $3 \mathrm{~mm}$ receivers (512 channels, $512 \mathrm{MHz}$ bandwidth, $1 \mathrm{MHz}$ channel spacing) and the $4 \mathrm{MHz}$ filter banks for the 2 and $1.3 \mathrm{~mm}$ observations (256 channels, $1 \mathrm{GHz}$ bandwidth, $4 \mathrm{MHz}$ channel spacing). The data were processed with the CLASS software. We omitted scans with distorted baselines, and only subtracted linear baselines from individual spectra. Their average was then regridded to a velocity resolution of $50 \mathrm{~km} \mathrm{~s}^{-1}$ (CO 3-2, 4-3, 5-4 ) and $55 \mathrm{~km} \mathrm{~s}^{-1}(6-5)$ leading to rms noise values $\left(T_{\mathrm{A}}^{*}\right)$ of $0.26 \mathrm{mK}(1.6 \mathrm{mJy}), 0.47 \mathrm{mK}$ (3.0 mJy), $0.6 \mathrm{mK}(4.1 \mathrm{mJy})$ and $0.38 \mathrm{mK}(2.9 \mathrm{mJy})$ respectively. The total observing time in the final spectra is $8.7 \mathrm{~h}$, $6.8 \mathrm{~h}, 4.7 \mathrm{~h}$ and $8.2 \mathrm{~h}$ for the 3-2 to 6-5 lines, respectively. The final spectra are presented in Fig. 1. We also generated an averaged $\mathrm{CO}$ spectrum by averaging all individual $\mathrm{CO}$ transitions, with equal weight. This spectrum is shown at a velocity resolution of $15 \mathrm{~km} \mathrm{~s}^{-1}$ in Fig. 2 .

\section{Results}

We detected all observed $\mathrm{CO}$ transitions from SMM J16359+6612 B. The line profiles for all four lines are similar, and show the characteristic double peak (see Fig. 1) already recognized in previous interferometric studies (Sheth et al. 2004; Kneib et al. 2005).

The small separation between the lensed images A and B of 15.0" (Kneib et al. 2004) implies that our spectra also have a contribution from component $\mathrm{A}$. Taking the relative integrated flux densities between component $\mathrm{A} / \mathrm{B}$ and the beam

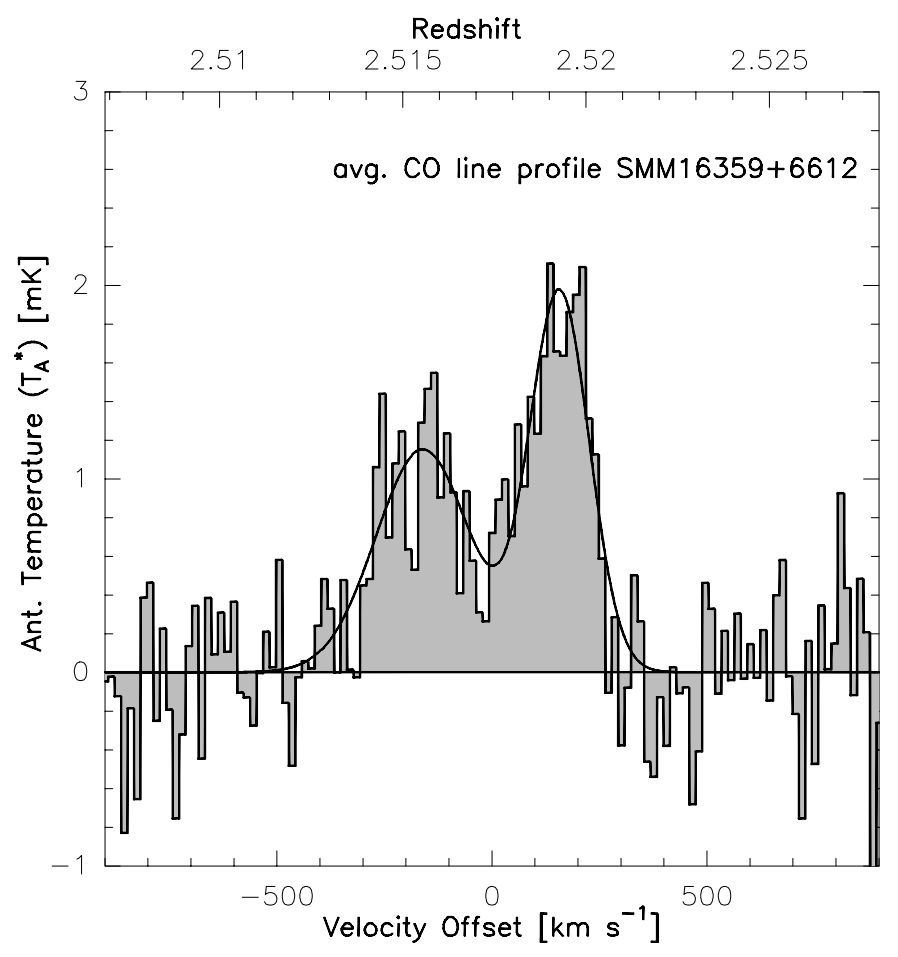

Fig. 2. Averaged spectrum of all four $\mathrm{CO}$ lines at a velocity resolution of $15 \mathrm{~km} \mathrm{~s}^{-1}$ with Gaussian fit profile superposed. All CO lines have equal weight. The velocity scale is relative to $z=2.5174$. The total integration time for the spectrum is 28.4 hours (on + off).

size of the $30 \mathrm{~m}$ telescope at the four observing frequencies into account, we estimate the contribution from component A to the integrated line intensities of our spectra to be $24 \%$, $12 \%, 4 \%$ and $2 \%$ for the $3-2$ to $6-5$ transitions respectively. These corrections have been applied to the following quantitative analysis. Due to the larger distance to component $C$ and its lower magnification, the contribution from this component is negligible.

Since the line profiles for all transitions are similar, we used the average spectrum of all CO lines (Fig. 2) to determine the line parameters of the blue and redshifted component in the spectrum. A Gaussian decomposition yields line widths of $250 \pm 45 \mathrm{~km} \mathrm{~s}^{-1}$ and $160 \pm 20 \mathrm{~km} \mathrm{~s}^{-1}$ for the blue and the red components respectively. The integrated flux density ratio between the blue and the red component is close to unity 
Table 1. Gaussian decomposition of the averaged CO line spectrum (see Fig. 2).

\begin{tabular}{lcc}
\hline \hline & Blue & Red \\
\hline$z$ & $2.5155 \pm 0.0002$ & $2.5192 \pm 0.0001$ \\
$\delta v\left[\mathrm{~km} \mathrm{~s}^{-1}\right]^{a}$ & $-161 \pm 17$ & $+156 \pm 9$ \\
$F W H M\left[\mathrm{~km} \mathrm{~s}^{-1}\right]$ & $250 \pm 45$ & $160 \pm 20$ \\
$S_{v \text { norm }}$ & $0.58 \pm 0.18$ & 1 \\
$I_{\text {norm }}$ & $0.91 \pm 0.16$ & 1 \\
\hline
\end{tabular}

${ }^{a}$ Center velocity relative to $z=2.5174$ (Kneib et al. 2005).

$\left(I_{B} / I_{R}=0.9 \pm 0.2\right)$. The parameters of the spectral decomposition are summarized in Table 1.

For the Gaussian decomposition of the individual CO lines we fixed the line width in both components to the values derived above. The line parameters for each transition are given in Table 2. Our integrated $\mathrm{CO}(3-2)$ line flux is in agreement with the Bure flux reported by Kneib etal. (2005) (2.8 vs. $2.5 \mathrm{Jy} \mathrm{km} \mathrm{s}^{-1}$ ) but lower than the flux reported by Sheth et al. (3.5 Jy $\mathrm{km} \mathrm{s}^{-1}$, 2004). The integrated and peak flux densities of the CO lines are rising with increasing rotational quantum number up to the 5-4 line. The line luminosities ( $L^{\prime}$, see e.g. Solomon et al. 1997, for the definition) show that already the 4-3 line is subthermally excited relative to the 3-2 line.

To investigate the $\mathrm{CO}$ excitation in more detail we used a spherical, one-component, large velocity gradient model (LVG). We use the collision rates from Flower (2001) with an ortho/para $\mathrm{H}_{2}$ ratio of 3 and a $\mathrm{CO}$ abundance per velocity gradient of $[\mathrm{CO}] / \mathrm{d} v / \mathrm{d} r=8 \times 10^{-5} \mathrm{pc}\left(\mathrm{km} \mathrm{s}^{-1}\right)^{-1}$. All lines are well fitted with a single LVG model. A good fit to the observations is provided by $n\left(\mathrm{H}_{2}\right)=10^{3.4} \mathrm{~cm}^{-3}$ and $T_{\text {kin }}=35 \mathrm{~K}$. Other temperature-density combinations with similar $\mathrm{H}_{2}$ pressure also match the data (see Fig. 3). $\mathrm{H}_{2}$ densities above $n\left(\mathrm{H}_{2}\right)=10^{3.8} \mathrm{~cm}^{-3}$, however, do not provide a good fit to the observations.

In Fig. 3 we compare selected LVG models with the observed integrated flux densities including the $\mathrm{CO} 3-2$ and 7-6 measurements by Kneib et al. (2005). We estimated the $7-6$ flux using a line ratio of $I_{\mathrm{CO}(7-6)} / I_{\mathrm{CO}(3-2)}=0.7$ (line integral corrected for the continuum emission at $1 \mathrm{~mm}$ ). The figure shows that the predicted integrated flux density for the $\mathrm{CO}(1-0)$ line is not a strong function of the model parameters. Our models predict $I_{\mathrm{CO}(1-0)} \approx 0.3-0.4 \mathrm{Jy} \mathrm{km} \mathrm{s}^{-1}$ or $L_{\mathrm{CO}(1-0)}^{\prime} \approx 4.1-5.6 \times 10^{9} \mathrm{~K} \mathrm{~km} \mathrm{~s}^{-1} \mathrm{pc}^{2}$ (corrected for a magnification $m=22$ ).

Our Gaussian decomposition of the individual CO transitions shows that the integrated flux density ratio between the blue and the red part of the spectrum is near unity for all $\mathrm{CO}$ transitions (see $I_{B} / I_{R}$ in Table 2). This implies that gas masses, $\mathrm{CO}$ line ratios and also the underlying excitation conditions are similar in the red and the blue components.

\section{Discussion}

At first glance the double horn line profile and the similarity of the gas properties in both spectral components suggests that we are looking at a circumnuclear molecular toroid. However, if such a stable molecular gas distribution would supply the large star formation rate $\left(\approx 500 M_{\odot} \mathrm{yr}^{-1}\right.$, Kneib et al. 2004) we would expect much higher gas excitation (the same is true if we were looking at independent molecular disks surrounding the nuclei of two merging galaxies). This is exemplified in Fig. 3 where we compare the normalized CO line SEDs (flux density vs. rotational quantum number) of SMM J16359+6612 to those in other starburst galaxies/QSOs. The normalization is relative to the integrated $\mathrm{CO}(1-0)$ flux density (and therefore to the molecular gas mass). These CO SEDs can be used to constrain the $\mathrm{CO}$ excitation as the turnover of the $\mathrm{CO}$ line SED is a sensitive measure of the underlying gas density and temperature.

In SMM J16359+6612 the CO SED turnover occurs at the 5-4 line, which shows that the average gas excitation is lower than in the center of the well-studied nearby starburst galaxies NGC 253 and M 82. In both cases, the CO SED turns over at the 6-5 line (Bradford et al. 2003; Bayet et al. 2004; Mao et al. 2000; Weiß et al. 2005). Even higher CO excitation is present in Henize 2-10 (Bayet et al. 2004) and some high- $z$ QSOs such as BR 1202-0725 $(z=4.7$, Omont et al. 1996; Carilli et al. 2002) and the Cloverleaf ( $z=2.6$, Barvainis et al. 1997) where the flux density is still rising even up to the $\mathrm{CO}(7-6)$ transition. This comparison shows that the molecular gas pressure in SMM J16359+6612 is lower than in other active systems. From our models, we conclude that the difference mainly arises due to the moderate average gas density $\left(n\left(\mathrm{H}_{2}\right)<10^{4} \mathrm{~cm}^{-3}\right)$ in SMM J16359+6612. This suggests that the molecular gas is less centrally concentrated than gas in starburst nuclei and QSO host galaxies. This view is supported by the large extent (1.5-3 kpc) of the molecular emission region found by Kneib et al. (2005).

One possiblity to explain the combination of lower CO excitation and high FIR luminosity $\left(10^{12} L_{\odot}\right.$, Kneib et al. 2004) may be that the CO flux comes from an "overlap" region between two merging galaxies. Such a geometry has first been suggested by Kneib et al. based on the UV/optical morphology of the potential two nuclei (their regions $\alpha$ and $\beta$ ) and the reddened overlap region (their region $\gamma$ ). Such active overlap regions are often found in nearby interacting systems (e.g., the Antennae: Wilson et al. 2000; NGC 6090: Bryant \& Scoville 1999; Wang et al. 2004).

For comparison, we therefore also show the CO SED for the overlap region in the Antennae in Fig. 3 (Zhu priv. com.). Although no observations above the $\mathrm{CO}(3-2)$ line have been published in literature so far, the low average $\mathrm{CO}(3-2) / \mathrm{CO}(1-0)$ line ratio suggests that the gas in the Antennae overlap region has a lower excitation than the starburst galaxies/QSOs and SMM J16359+6612. More detailed models of the molecular gas in the Antennae show that the bulk of the $\mathrm{CO}$ emission arises from gas at moderate density of $n\left(\mathrm{H}_{2}\right) \approx 10^{3} \mathrm{~cm}^{-3}$ (Zhu et al. 2003). Given the early stage of the merging process of the Antennae and its low FIR luminosity $\left(\sim 5 \times 10^{10} L_{\odot}\right.$, Sanders et al. 2003) we suggest that SMM J16359+6612 may be a more advanced merger (with higher FIR luminosity and higher mean gas density than in the Antennae overlap region) but not yet in the typical ULIRG 
Table 2. CO line parameters towards SMMJ16359+6612 B, corrected for the contribution from image A. The mean redshift is the fluxweighted average of the redshift of the red $(R)$ and blue $(B)$ component. $\delta V_{R, B}$ denotes the peak-to-peak velocity separation between the red and blue components. Parameters have been derived from Gaussian fits by keeping the line widths fixed at 250 and $160 \mathrm{~km} \mathrm{~s}^{-1}$ for the blue and red components.

\begin{tabular}{lcrcccccr}
\hline \hline Transition & $z_{\text {mean }}$ & $\begin{array}{c}S_{v R} \\
{[\mathrm{mJy}]}\end{array}$ & $\begin{array}{c}S_{v B} \\
{[\mathrm{mJy}]}\end{array}$ & $\begin{array}{c}\delta V_{R, B} \\
{\left[\mathrm{~km} \mathrm{~s}^{-1}\right]}\end{array}$ & $\begin{array}{c}I \\
{\left[\mathrm{Jy} \mathrm{km} \mathrm{s} \mathrm{km}^{-1}\right]}\end{array}$ & $\begin{array}{c}I_{B} / I_{R} \\
{\left[10^{9} \mathrm{~K} \mathrm{~km} \mathrm{~s}^{-1} \mathrm{pc}^{2}\right]}\end{array}$ & $\begin{array}{c}L^{a} \\
{\left[10^{6} L_{\odot}\right]}\end{array}$ \\
\hline $\mathrm{CO}(J=3 \rightarrow 2)$ & 2.51737 & $8.5 \pm 0.7$ & $5.3 \pm 0.6$ & $310 \pm 20$ & $2.8 \pm 0.2$ & $1.0 \pm 0.1$ & $4.4 \pm 0.3$ & $5.8 \pm 0.4$ \\
$\mathrm{CO}(J=4 \rightarrow 3)$ & 2.51732 & $11.9 \pm 1.5$ & $7.3 \pm 1.2$ & $340 \pm 25$ & $4.0 \pm 0.4$ & $1.0 \pm 0.2$ & $3.4 \pm 0.3$ & $10.7 \pm 1.1$ \\
$\mathrm{CO}(J=5 \rightarrow 4)$ & 2.51746 & $15.1 \pm 2.0$ & $9.7 \pm 1.6$ & $295 \pm 30$ & $5.1 \pm 0.5$ & $1.0 \pm 0.2$ & $2.8 \pm 0.3$ & $17.3 \pm 1.8$ \\
$\mathrm{CO}(J=6 \rightarrow 5)$ & 2.51738 & $12.5 \pm 1.9$ & $7.0 \pm 1.5$ & $340 \pm 35$ & $4.0 \pm 0.5$ & $0.9 \pm 0.2$ & $1.5 \pm 0.2$ & $16.1 \pm 2.1$ \\
\hline
\end{tabular}

${ }^{a}$ Corrected for a magnification $m=22$ (Kneib et al. 2004).
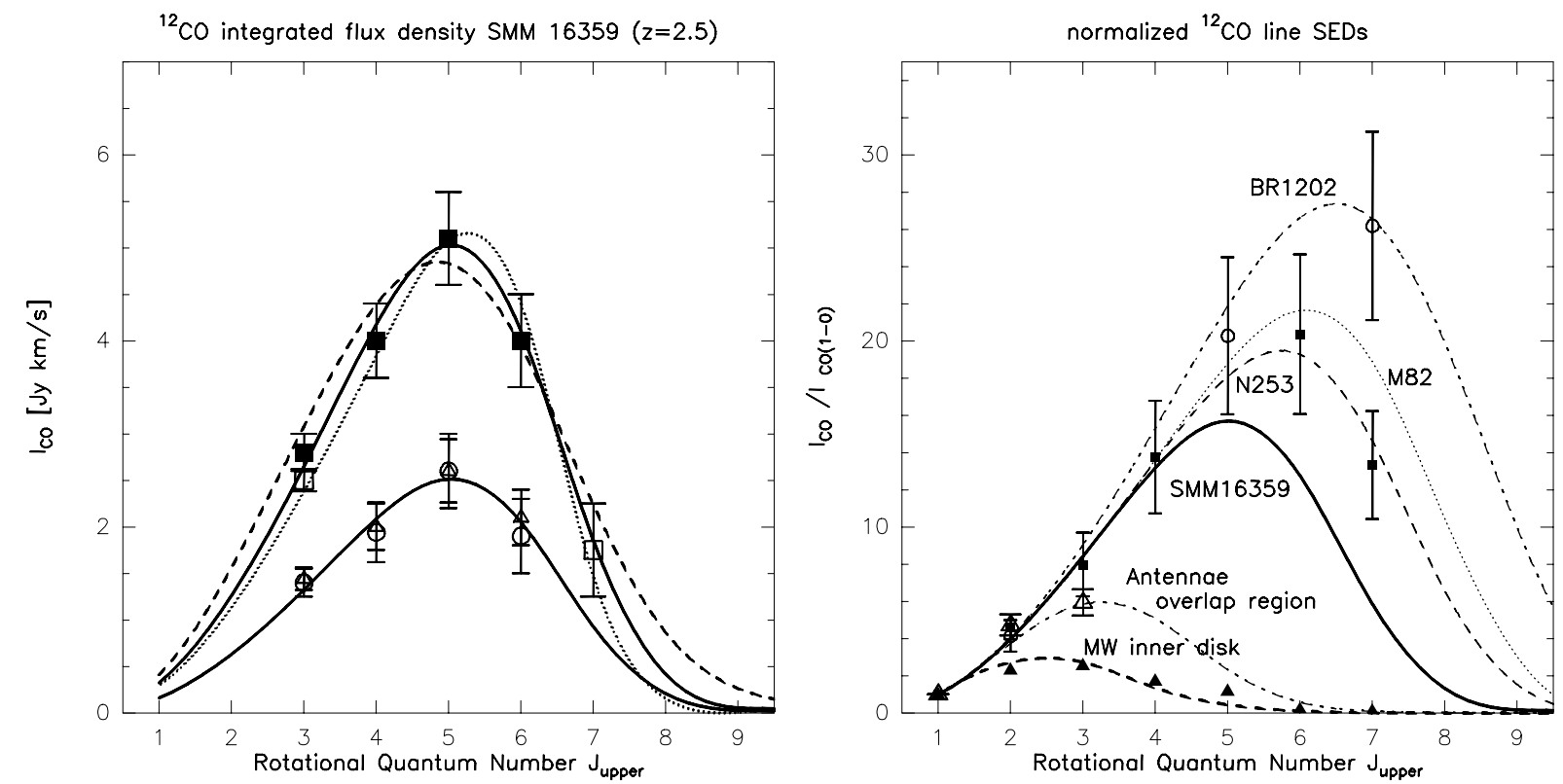

Fig. 3. Left: observed, velocity-integrated CO fluxes vs. rotational quantum number (CO line SED). Filled squares are the integrated flux densities listed in Table 2, open squares were adopted from Kneib et al. (2005). The upper three curves are LVG models for the entire line profile (red+blue components added together). The lines show the LVG-predicted fluxes for $n\left(\mathrm{H}_{2}\right), T_{\text {kin }}$ of $10^{3.4} \mathrm{~cm}^{-3}, 35 \mathrm{~K}(\mathrm{solid}), 10^{3.1} \mathrm{~cm}^{-3}$, $80 \mathrm{~K}$ (dashed) and $10^{3.8} \mathrm{~cm}^{-3}, 20 \mathrm{~K}$ (dotted). The lower data points are the fluxes for the red (open triangles) and blue (open circles) components taken separately. The lower curve shows the same LVG model as the solid upper curve. Right: comparison of various normalized CO line SEDs: SEDs are shown for BR 1202-0725 $(z=4.7$, open circles Carilli et al. 2002), the high-excitation component in the center of M 82 (Weiß et al. 2005, no data points plotted), NGC 253 center (filled squares taken from Bayet et al. 2004), SMM J16359+6612 (no data points plotted), the overlap region in the Antennae galaxies (open triangles Zhu et al. 2003) and the inner disk of the Milky Way (filled triangles, Fixsen et al. 1999). The CO line SEDs are normalized by their CO(1-0) flux density.

stage (with the gas concentrated in the central $<1 \mathrm{kpc}$ region), such as, e.g., Mrk 231.

Acknowledgements. We thank M. Zhu for providing us with the CO fluxes for the Antennae and the IRAM receiver engineers D. John and S. Navarro as well as the telescope operators for their great support on optimizing the receiver tuning. IRAM is supported by INSU/CNRS (France), MPG (Germany) and IGN (Spain).

\section{References}

Barvainis, R., Maloney, P., Antonucci, R., \& Alloin, D. 1997, ApJ, 484,695

Bayet, E., Gerin, M., Phillips, T. G., \& Contursi, A. 2004, A\&A, 427, 45
Bertoldi, F., Carilli, C. L., Menten, K. M., et al. 2000, A\&A, 360, 92 Blain, A. W., Smail, I., Ivison, R. J., Kneib, J. P., \& Frayer, D. T. 2002, Phys. Rep., 369, 111

Bradford, C. M., Nikola, T., Stacey, C. J., et al. 2003, ApJ, 586, 891

Bryant, P. M., \& Scoville, N. Z. 1999, AJ, 117, 2632

Carilli, C. L., Kohno, K., Kawabe, R., et al. 2002, AJ, 123, 1838

Downes, D., \& Solomon, P. M. 1998, ApJ, 507, 615

Downes, D., \& Solomon, P. M. 2003, ApJ, 528, 37

Fixsen, D. J., Dwek, E., Mather, J. C., Bennett, C. L., \& Shafer, R. A. 1998, ApJ, 508, 123

Fixsen, D. J., Bennett, C. L., \& Mather, J. C. 1999, ApJ, 526, 207

Flower, D. R. 2001, J. Phys. B: At. Mol. Opt. Phys., 34, 1

Frayer, D. T., Ivison, R., Scoville, N. Z., et al. 1998, ApJ, 506, L7

Genzel, R., Baker, A., Tacconi, L., et al. 2003, ApJ, 584, 633

Greve, T. R., Ivison, R. J., Bertoldi, F., et al. 2004, MNRAS, 354, 779 
Greve, T. R., Bertoldi, F., Smail, I., et al. 2005, MNRAS, 359, 1165 Ivison, R. J., Smail, I., Frayer, D. T., Kneib, J. P., \& Blain, A. W. 2001, ApJ, 561, L45

Ivison, R. J., Greve, T. R., Smail, I., et al. 2002, MNRAS, 337, 1

Kneib, J.-P., van der Werf, P., Knudsen, K. K., et al. 2004, MNRAS, 349,1211

Kneib, J.-P., Neri, R., Smail, I., et al. 2005, A\&A, 434, 819

Mao, R. Q., Henkel, C., Schulz, A., et al. 2000, A\&A, 358, 433

Neri, R., Genzel, R., Ivison, R. J., et al. 2003, ApJ, 597, L113

Omont, A., Petitjean, P., Guilloteau, S., et al. 1996, Nature, 382, 428

Puget, J.-L., Abergel, A., Bernard, J.-P., et al. 1996, A\&A, 308, 5

Sanders, D. B., Mazzarella, J. M., Kim, D.-C., Surace, J. A., \& Soifer, B. T. 2003, AJ, 126, 1607
Sheth, K., Blain, A., Kneib, J.-P., et al. 2004, ApJ, 614, L5

Smail, I., Ivison, R. J., \& Blain, A. 1997, ApJ, 490, L5

Solomon, P. M., Downes, D., Radford, S. J. E., \& Barrett, W. J. 1997, ApJ, 478, 144

Spergel, D. N., Verde, L., Peiris, H., et al. 2003, ApJS, 148, 175

Wang, J., Zhang, Q., Wang, Z., Ho, P. T. P., Fazio, G. G., \& Wu, Y. 2004, ApJ, 616, 67

Webb, T. M. A., Lilly, S. J., Clemens, D. L., et al. 2003, ApJ, 597, 680 Weiß, A., Walter, F., \& Scoville, N. Z. 2005, A\&A, 438, 533

Wilson, C. D., Scoville, N., Madden, S. C., \& Charmandaris, V. 2000, ApJ, 542, 120

Zhu, M., Seaquist, E. R., \& Kuno, N. 2003, ApJ, 588, 243 